\title{
Towards Understanding the Origin of Cosmic-Ray Electrons
}

\section{Cheng ZHANG ${ }^{* \dagger}$}

Institute of High Energy Physics, CAS, Beijing, China

E-mail: cheng.zhang@cern.ch

Precision results on cosmic-ray electrons, based on $28.1 \times 10^{6}$ electrons collected by the Alpha Magnetic Spectrometer on the International Space Station, are presented in the energy range from $0.5 \mathrm{GeV}$ to $1.4 \mathrm{TeV}$. Compared to the positron spectrum, the electron spectrum has distinctly different magnitudes and energy dependences in the entire energy range. At low energy region, electron flux exhibits a significant excess starting from $42.1_{-5.2}^{+5.4} \mathrm{GeV}$ compared to the lower energy trends, but the nature of this excess is different from the positron flux excess above $25.2 \pm 1.8$ $\mathrm{GeV}$. At high energy region, it does not have an energy cutoff below $1.9 \mathrm{TeV}$ at the $5 \sigma$ level, which is contrary to the positron flux with an exponential energy cutoff of $810_{-180}^{+310} \mathrm{GeV}$. In the entire energy range the electron flux is well described by the sum of two power law components. It's a clear evidence that most high energy electrons originate from different sources than high energy positrons, based on the different behavior of the cosmic-ray electrons and positrons measured by the Alpha Magnetic Spectrometer.

European Physical Society Conference on High Energy Physics - EPS-HEP2019 -

10-17 July, 2019

Ghent, Belgium

\footnotetext{
* Speaker.

${ }^{\dagger}$ on behalf of the AMS Collaboration
} 
The detailed studies of positron spectrum up to $1 \mathrm{TeV}$ also has been performed by AMS[1,2]. The observed new source of high energy positrons poses the question of the existence of a similar source in cosmic-ray electrons. Many models could explain the AMS results on cosmic-ray electrons and positrons, which fall into three distinct classes: annihilation of dark matter particles[3], production in the interactions of cosmic-ray nuclei with interstellar gas[4], and acceleration to high energies in astrophysical objects[5]. The difference for most of these explanations is in their predictions for the behavior of cosmic-ray electrons and positrons at high energies.

In this proceeding we present precision measurements of primary cosmic-ray electrons up to 1.4 $\mathrm{TeV}[6]$ and the comparison with our latest data on cosmic ray positrons[2]. The electron flux is measured with the Alpha Magnetic Spectrometer (AMS) on the International Space Station (ISS), which is based on $28.1 \times 10^{6}$ electron events collected from May 19, 2011 to November 12, 2017. It has a factor of 3 increase in statistics and a factor of 2 increase in the energy range compared to our results published nearly five years ago[7]. The AMS observation of distinctive properties of electron and positron fluxes are crucial for providing insights into origins of high energy cosmic-ray electrons and positrons.

\section{AMS-02 Detector}

The full description of the AMS detector is presented in Ref.[6] and references therein. The key detector elements used for the electron analysis are the transition radiation detector TRD, the time of flight counters TOF, the silicon tracker, the permanent magnet, and the electromagnetic calorimeter ECAL.

The tracker has nine layers, the first L1 at the top of the detector, the second L2 above the permanent magnet, L3 to L8 within the bore of the magnet, and the last L9 above the ECAL. The nine-layer tracker accurately measures the rigidity $\mathrm{R}$ and the charge $\mathrm{Z}$ of cosmic rays. For $\mid \mathrm{ZI}=1$ particles the maximum detectable rigidity is $2 \mathrm{TV}$ over the 3 meters lever arm and the charge resolution is $\Delta \mathrm{Z}=0.05$. The TOF measures $|\mathrm{Z}|$ with a resolution $\Delta \mathrm{Z}=0.05$ and velocity $\beta$ with $\Delta \beta / \beta^{2}=4 \%$. The TRD separates electrons and positrons $e^{ \pm}$from protons $\mathrm{p}$ with a $\Lambda_{T R D}$ estimator[1], which is constructed from the ratio of the log-likelihood probability of the $e^{ \pm}$hypothesis to that of the $\mathrm{p}$ hypothesis in each layer of the 20 layers proportional tubes. The ECAL has 17 radiation length. It could accurately measure the $e^{ \pm}$energies and shower shape with the three-dimensional imaging capability. The $\Lambda_{E C A L}$ estimator[8] is used to differentiate $e^{ \pm}$from $\mathrm{p}$ by exploiting their different shower shapes.

The charge confusion estimator $\Lambda_{C C}^{e}[2,6,9]$ is used to distinguish electrons from charge confusion positrons and protons, that is, positron and proton events reconstructed in the tracker with negative rigidity due to the finite tracker resolution or due to interactions with the detector materials. $\Lambda_{C C}^{e}$ is efficient for the sparation between electron signal and charge confusion background in data analysis.

The entire detector has been extensively calibrated in a test beam at CERN with measurements in 18 different energies and particles at 2000 positions. That includes $e^{+}$and $e^{-}$from 10 to 290 $\mathrm{GeV} / \mathrm{c}, \mathrm{p}$ at 180 and $400 \mathrm{GeV} / \mathrm{c}$, and $\pi$ from 10 to $180 \mathrm{GeV} / \mathrm{c}$, which produce transition radiation equivalent to $\mathrm{p}$ up to $1.2 \mathrm{TeV} / \mathrm{c}$. On orbit, the cosmic ray proton data is used to extend the calibration 
to higher energy and the detector performance is continuously monitored and keeps steady over time.

A Monte Carlo program based on the GEANT4 10.1 package[10] is used to simulate physics processes and signals in the detector. It's developed based on the results of extensive calibrations both on the ground and in space, and provides an excellent description of the data.

\section{Electron Measurement}

The detailed analysis procedure for electron flux measurement is described in Ref.[6]. After event selections, the negative rigidity event sample is comprised of mostly electron signal events and a small amount of antiproton, charge confusion positron and proton background events. The combination of information from the TRD, tracker, and ECAL enables the efficient separation of the electron signal events from background events using a template fitting technique. In the energy region [0.5-1000] GeV an energy-dependent cut on ECAL estimator $\Lambda_{E C A L}$ is applied to remove the bulk of the antiproton and charge confusion proton background. The number of electrons and its statistical error in each bin are then determined by fitting signal and background templates to data in the two dimensional variable space of $\left(\Lambda_{T R D}-\Lambda_{C C}^{e}\right)$ by varying their respective normalizations. In the energy bin [1000-1400] GeV the number of electrons is determined by fitting the $\Lambda_{E C A L}$ template, which seperates electron signal from the antiproton and charge confusion proton background. The amount of the charge confusion positron background is estimated from the Monte Carlo simulation because of the same shape of $\Lambda_{E C A L}$ for electrons and charge confusion positrons. In total, $28.1 \times 10^{6}$ electrons are identified in the energy range from $0.5 \mathrm{GeV}$ to $1.4 \mathrm{TeV}$.

The isotropic electron flux for the energy bin $E_{i}$ of width $\Delta E_{i}$ at the top of AMS is calculated by:

$$
\Phi_{e^{-}, i}=\frac{N_{i}}{A_{i}\left(1+\delta_{i}\right) T_{i} \Delta E_{i}}
$$

where $N_{i}$ is the number of $e^{-}$in the energy bin $i$ corrected for the small bin-to-bin migration using the unfolding procedure described in Ref.[11]. $A_{i}$ is the effective acceptance calculated from MC simulation. $T_{i}$ is the data collection time. $\delta_{i}$ is minute corrections estimated by comparing the efficiencies in data and MC simulation of every selection cut using information from the detectors unrelated to that cut.

Detailed study of the systematic errors is key part of the analysis. Systematic uncertainties for the electron flux include uncertainties from: template definition, charge confusion determination, efficiency correction $\delta_{i}$, bin-to-bin migration and energy scale. The template definition uncertainty includes two parts: the event selection and the statistical fluctuation. The associated systematic error of event selection is $<0.3 \%$ in the entire energy range. The associated error of fluctuation is $<0.4 \%$ below $500 \mathrm{GeV}, \leq 2 \%$ below $1000 \mathrm{GeV}$, and $3.5 \%$ in the last bin[1000-1400]GeV. The magnitude of the charge confusion uncertainty accounts for the small differences between data and MC simulation. It's $\leq 0.6 \%$ of the flux below $500 \mathrm{GeV}, \leq 2 \%$ blow $1000 \mathrm{GeV}$, and $3 \%$ in the last energy bin [1000-1400]GeV. The uncertainty in the efficiency correction is $4 \%$ at $0.5 \mathrm{GeV}$, decreases to $1.1 \%$ at $3 \mathrm{GeV}$ and slowly rises up to $2.5 \%$ in the last bin [1000-1400] GeV. The correlated systematic error on the flux normalization is included in the efficiency correction uncertainty, which is estimated to be $1 \%$ and is subtracted in quadrature from the total systematic error. The bin-to-bin 
migration uncertainty due to the finite energy resolution is small. It's $2 \%$ at $0.5 \mathrm{GeV}$ and decreases to $<0.2 \%$ above $10 \mathrm{GeV}$. The energy scale error is $4 \%$ at $0.5 \mathrm{GeV}, 2 \%$ from 2 to $300 \mathrm{GeV}$, and $2.6 \%$ at $1.4 \mathrm{TeV}$. The detail of it has been discussed in Ref.[8]

The uncertainties in template definition, charge confusion, the efficiency corrections and binto-bin migration are added in quadrature to get the total systematic error of the electron flux. The energy scale error is treated as an uncertainty of the bin boundaries. The detailed discussion about the study of systematic errors is in Ref.[6].

Most importantly, statistical error becomes dominating the total error above $200 \mathrm{GeV}$ for electron flux measurment. Therefore by continuing taking data, AMS will be able to improve the accuracy of the measurement and reach into uncharted high energy range.

\section{Distinctive Properties of Electron Flux}

Fig. 1 shows the measured AMS electron spectrum in comparison with the most recent AMS positron spectrum[2] scaled by a factor of 10, and the error bars correspond to the quadratic sum of statistical and systematic errors. For display purpose, both the electron and positron spectra are presented scaled by $\tilde{E}^{3}$. As seen, the electron and positron spectra have distinctly different magnitudes and energy dependences. Several checks with tighter selection criteria were performed on the same negative rigidity data sample and yielded consistent results.

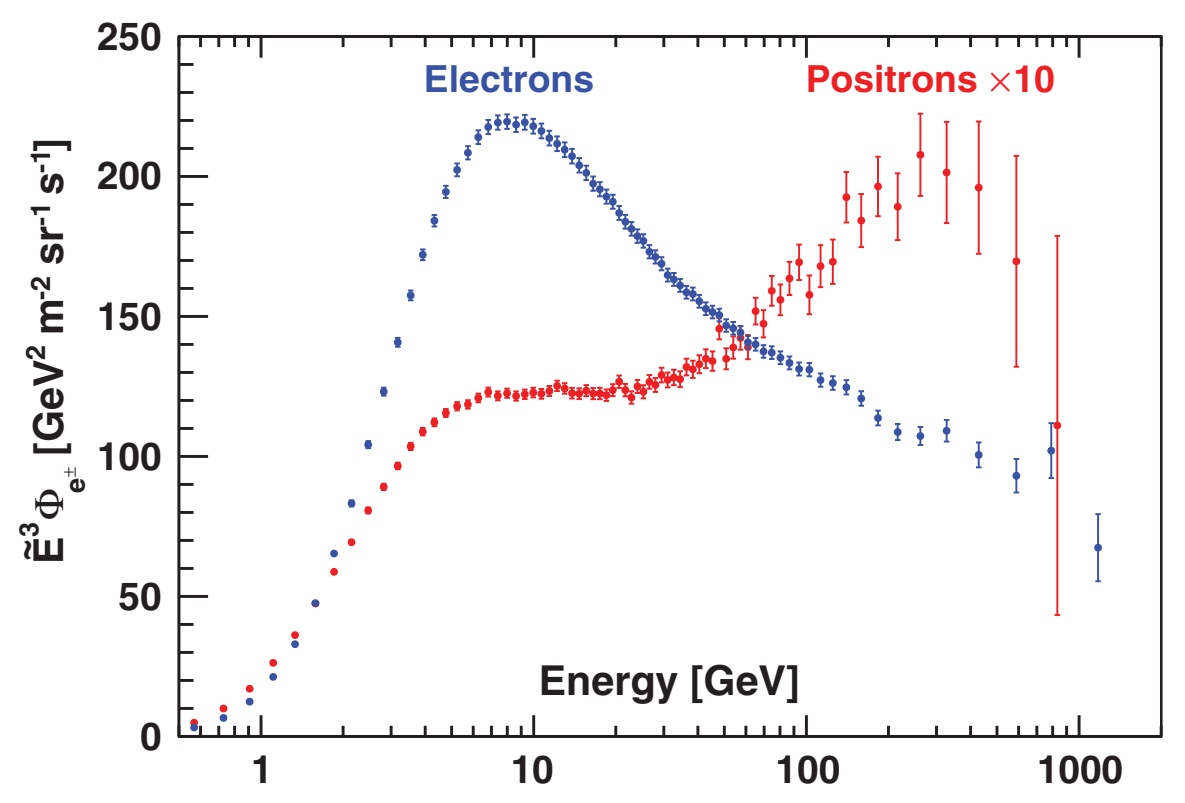

Figure 1: The AMS electron (blue data points) and positron (red data points, multiplied by 10) spectra $\left(\tilde{E}^{3} \Phi_{e^{ \pm}}\right)$. For display purposes the electron data point at $\sim 830 \mathrm{GeV}$ is slightly shifted horizontally to avoid overlap with the positron point. As seen, the electron spectrum has distinctly different magnitude and energy dependence compared to that of positron spectrum.

To study the energy dependence of the electron flux in a model-independent way, the flux spectral index $\gamma$ is calculated from

$$
\gamma=d[\log (\Phi)] / d[\log (E)]
$$


over non-overlapping energy intervals. The AMS electron flux spectral indices are presented in Fig. 2 compared with the AMS positron results[2]. As seen in figure, below $\sim 10 \mathrm{GeV}$, both the electron and positron indices decrease (soften) rapidly with energy, and then above $20 \mathrm{GeV}$, they both start increasing (harden). Particularly, the electron spectral index increases from $\gamma=-3.295$ \pm 0.026 in the energy range [17.98-27.25] GeV to an average $\gamma=-3.180 \pm 0.008$ in the range [55.58-1400] GeV, where it is nearly energy independent. As seen in Fig.2, the behavior of the electron and positron spectral indices is distinctly different.

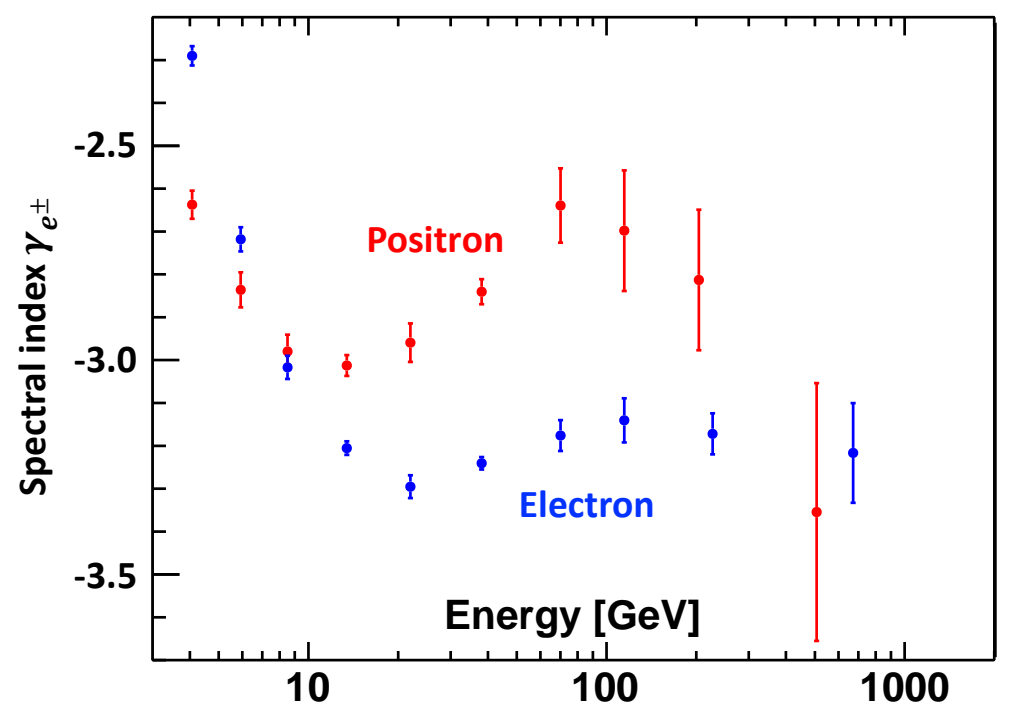

Figure 2: The spectral indices of the AMS electron (blue data points) and positron[2] (red data points) spectra in non-overlapping energy intervals. They show distinctly different behavior.

To determine the transition energy $E_{0}$ where the change of the electron spectral index occurs, a double power law approximation is used:

$$
\Phi_{e^{-}}(E)= \begin{cases}C(E / 20.04 \mathrm{GeV})^{\gamma}, & E \leq E_{0} \\ C(E / 20.04 \mathrm{GeV})^{\gamma}\left(E / E_{0}\right)^{\Delta \gamma}, & E>E_{0}\end{cases}
$$

A fit to data in the energy range [20.04-1400] GeV is presented in Fig.3. The fit yields $E_{0}=42.1_{-5.2}^{+5.4} \mathrm{GeV}$ for the energy where the spectral index increases with $C=2.335_{-0.019}^{+0.014} \times$ $10^{-2}\left[\mathrm{~m}^{2} \text { sr s GeV }\right]^{-1}, \gamma=-3.280_{-0.016}^{+0.014}, \Delta \gamma=0.094 \pm 0.014$, and $\chi^{2} /$ d.o.f. $=17.9 / 36$. The energy $E_{0}$ corresponds to the beginning of a significant excess of the electron flux compared to the lower energy trends. The spectral index change by $\Delta \gamma$ is clearly visible in Fig.3.

For the sources of primary cosmic-ray electrons, there are several astrophysical sources and it's assumed that there are only a few astrophysical sources of high energy electrons in the vicinity of the Solar System each making a power-law-like contribution to the electron flux[12]. In addition, several physics effects may introduce some spectral features in the original fluxes [13, 14] and the collisions of ordinary cosmic rays with the interstellar gas[15] has a small contribution of secondary electrons. Therefore, the minimal number of distinct power law functions needed to accurately describe the AMS electron flux is important to be known. 


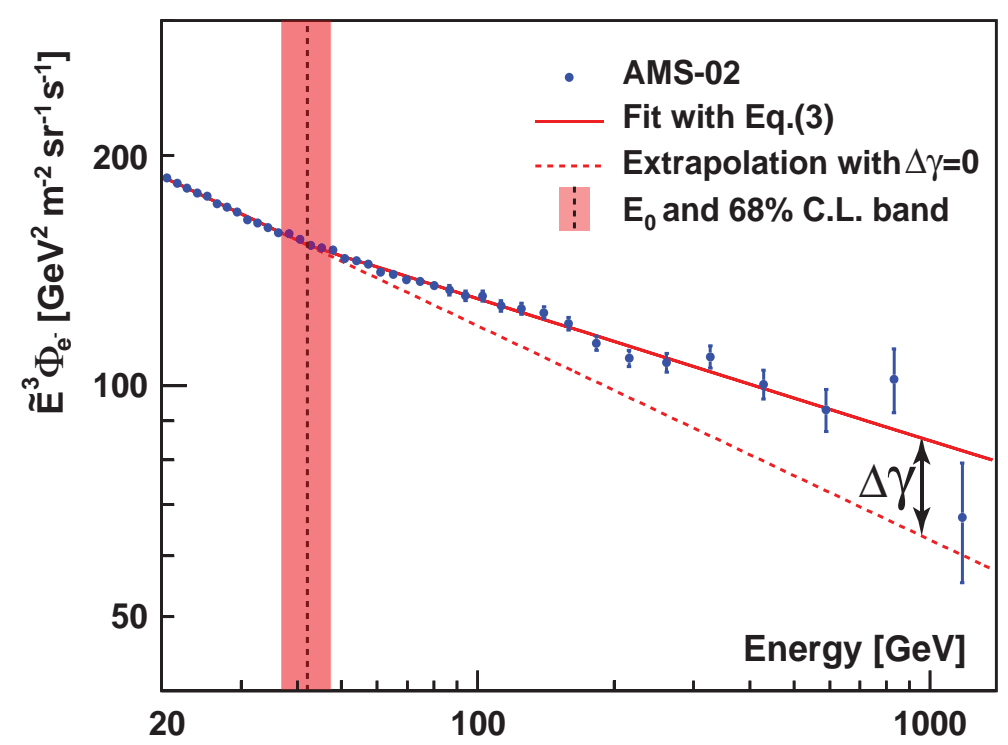

Figure 3: A double power law fit of Eq.(3) to the electron flux in the energy range [20.04-1400] GeV. The blue data points are the measured electron flux scaled by $\tilde{E}^{3}$. The solid red line is the fitted function. The vertical dashed line and the red band correspond to the value and the error of the energy $E_{0}$ where the change of the spectral index occurs and $\Delta \gamma=0.094 \pm 0.014$ is the magnitude of the spectral index change; as seen in Eq.(3). The dashed red line is the extrapolation of the power law below $E_{0}$ into the higher energy range [or $\Delta \gamma=0$ in Eq.(3) ].

In the entire energy range [0.5-1400] GeV, it's found that the sum of two power law components could well describe the electron flux:

$$
\Phi_{e^{-}}(E)=\frac{E^{2}}{\hat{E}^{2}}\left[1+\left(\hat{E} / E_{t}\right)^{\Delta \gamma_{t}}\right]^{-1}\left[C_{a}\left(\hat{E} / E_{a}\right)^{\gamma_{a}}+C_{b}\left(\hat{E} / E_{b}\right)^{\gamma_{b}}\right] .
$$

The additional transition term $\left[1+\left(\hat{E} / E_{t}\right)^{\Delta \gamma_{t}}\right]^{-1}$ is introduced to account for the effects related to the complex spectral behavior of the electron flux below $\sim 10 \mathrm{GeV}[13,16]$. The two power law function components, a and b, correspond to normalization factors $C_{a}$ and $C_{b}$, and spectral indices $\gamma_{a}$ and $\gamma_{b}$ respectively. The force-field approximation[17] is used to account for solar modulation effect such that the energy of particles in the interstellar space $\hat{E}=\mathrm{E}+\varphi_{e^{-}}$, where $\varphi_{e^{-}}$is the effective solar potential. The constant $E_{a}$ and $E_{b}$ does not affect the shapes nor the magnitudes of the two contribution and are chosen to be $20 \mathrm{GeV}$ and $300 \mathrm{GeV}$ respectively to minimize correlation between parameters. The fit of Eq.(4) to the measured flux yields $C_{a}=(1.13 \pm 0.08)$ $\times 10^{-2}\left[\mathrm{~m}^{2} \mathrm{sr} \mathrm{s} \mathrm{GeV}\right]^{-1}, \gamma_{a}=-4.31 \pm 0.13$ for the power law a, and $C_{b}=(3.96 \pm 0.04) \times 10^{-6}$ $\left[\mathrm{m}^{2} \mathrm{sr} \mathrm{s} \mathrm{GeV}\right]^{-1}, \gamma_{b}=-3.14 \pm 0.02$ for the power law $\mathrm{b}$ and $\chi^{2} /$ d.o.f. $=36.5 / 68$. Other fitted parameters are: $\varphi_{e^{-}}=0.87 \pm 0.12 \mathrm{GeV}$ for the effective potential, $E_{t}=3.94 \pm 0.21 \mathrm{GeV}$ and $\Delta \gamma_{t}=$ $-2.14 \pm 0.09$ for the transition term parameters.

The fit result is presented in Fig.4 and the sum of two power law functions with the additional transition term provides an excellent description of the data in the entire energy range [0.5-1400] $\mathrm{GeV}$. As seen, the two power law functions are very different in shape and in magnitude from those describing the positron flux[2]. Contrary to the interstellar secondary production term in positrons, which dominates the positron flux below $10 \mathrm{GeV}$, the contribution of power law a exceeds 


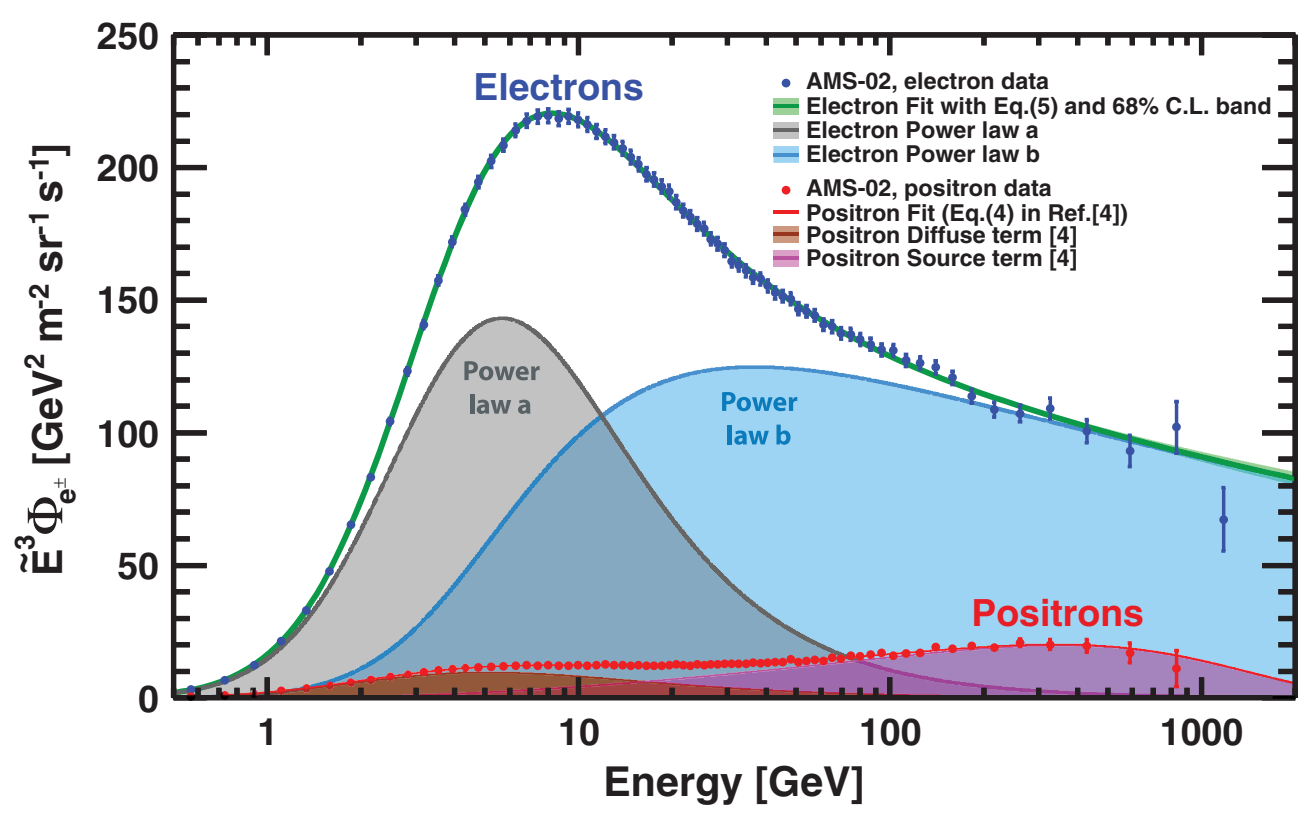

Figure 4: The two power law fit of Eq.(4) to the electron flux data in the energy range [0.5-1400] GeV with $68 \%$ C.L. (green band). The two power law components a and b of Eq.(4) are shown as the gray and blue areas, respectively. Also shown are the positron spectrum together with the fit function of Ref.[2] including the positron diffuse (i.e., interstellar secondary production) term (the brown area) and positron source term (the magenta area) contributions.

the expected secondary electron or positron production by a factor of $\sim 20$ (see Ref.[15]). The contribution of power law b, which dominates the electron flux at high energies above $40 \mathrm{GeV}$, significantly exceeds the magnitude of the positron source term [2], which has an exponential energy cutoff at $810_{-180}^{+310} \mathrm{GeV}$ (as seen in Fig.4). At $5 \sigma$ level, the electron flux does not have an energy cutoff below $1.9 \mathrm{TeV}$. Accordingly, the excess of the electron flux at $E_{0}=42.1_{-5.2}^{+5.4} \mathrm{GeV}$ compared to the lower energy trends has a different nature compared to positron flux excess at 25.2 $\pm 1.8 \mathrm{GeV}$. This is clear evidence that most cosmic-ray electrons originate from different sources than cosmic-ray positrons.

\section{Conclusion}

Based on $28.1 \times 10^{6}$ cosmic-ray electron events, we have presented the precision measurements of the electron flux from $0.5 \mathrm{GeV}$ to $1.4 \mathrm{TeV}$ with detailed study of systematic uncertainties. Compared to the AMS positron flux, AMS electron flux has distinctly different magnitudes and energy dependences in the entire energy range and is well described by the sum of two power law components. Above $42.1_{-5.2}^{+5.4} \mathrm{GeV}$ the electron flux has a significant excess compared to the low energy trends, which has a different nature compares to the positron flux excess starting from 25.2 $\pm 1.8 \mathrm{GeV}$. The electron flux does not exhibit an energy cutoff below $1.9 \mathrm{TeV}$ at the $5 \sigma$ level, but for the positron flux, there is an exponential energy cutoff of $810_{-180}^{+310} \mathrm{GeV}$. The different behavior of the cosmic-ray electrons and positrons measured by AMS is clear evidence that most high energy electrons originate from different sources than high energy positrons. 


\section{References}

[1] M. Aguilar et al., Phys. Rev. Lett. 110, 141102 (2013);L. Accardo et al., Phys. Rev. Lett. 113, 121101 (2014).

[2] M. Aguilar et al., Phys. Rev. Lett. 122, 041102 (2019).

[3] M. S. Turner and F. Wilczek, Phys. Rev. D 42, 1001 (1990); J. Ellis, AIP Conf. Proc. 516, 21 (2000); H. C. Cheng, J. L. Feng, and K. T. Matchev, Phys. Rev. Lett. 89, 211301(2002); G. Kane, R. Lu, and S. Watson, Phys. Lett. B 681,151 (2009); J. Kopp, Phys. Rev. D 88, 076013 (2013); C. H. Chen, C. W. Chiang, and T. Nomura, Phys. Lett. B 747, 495 (2015); H. C. Cheng, W. C. Huang, X. Huang, I. Low, Y. L. Sming Tsia, and Q. Yuan, J. Cosmol. Astropart. Phys. 03(2017) 041; S. J. Lin, Q. Yuan, and X. J. Bi, Phys. Rev. D91, 063508 (2015); Y. Bai, J. Berger, and S. Lu, Phys. Rev.D 97, 115012 (2018).

[4] R. Cowsik, B. Burch, and T. Madziwa-Nussinov, Astrophys.J. 786, 124 (2014); K. Blum, B. Katz, and E. Waxman, Phys. Rev. Lett. 111, 211101 (2013).

[5] P. D. Serpico, Astropart. Phys. 39-40, 2 (2012); T. Linden and S. Profumo, Astrophys. J. 772, 18 (2013); P. Mertsch and S. Sarkar, Phys. Rev. D 90, 061301 (2014); M. Di Mauro, F. Donato, N. Fornengo, R. Lineros, and A. Vittino, J. Cosmol. Astropart. Phys. 04 (2014) 006; N.Tomassetti and F. Donato, Astrophys. J. Lett. 803, L15 (2015); D. Hooper, I. Cholis, T. Linden, and K. Fang, Phys. Rev. D 96, 103013 (2017); W. Liu, X. J. Bi, S. J. Lin, B. B. Wang, and P. F. Yin, Phys. Rev. D 96, 023006 (2017); S. Profumo, J. Reynoso-Cordova, N. Kaaz, and M. Silverman, Phys. Rev. D 97, 123008 (2018).

[6] M. Aguilar et al., Phys. Rev. Lett. 122, 101101 (2019).

[7] M. Aguilar et al., Phys. Rev. Lett. 113, 121102 (2014).

[8] A. Kounine, Z. Weng, W. Xu, and C. Zhang, Nucl. Instrum. Methods Phys. Res., Sect. A 869, 110 (2017).

[9] M. Aguilar et al., Phys. Rev. Lett. 117, 091103 (2016).

[10] J. Allison et al., Nucl. Instrum. Methods Phys. Res., Sect. A835, 186 (2016); S. Agostinelli et al., Nucl. Instrum. Methods Phys. Res., Sect. A 506, 250 (2003).

[11] M. Aguilar et al., Phys. Rev. Lett. 114, 171103 (2015).

[12] A. M. Hillas, J. Phys. G 31, R95 (2005); Y. Z. Fan, B. Zhang, and J. Chang, Int. J. Mod. Phys. D 19, 2011 (2010). T. Kobayashi, Y. Komori, K. Yoshida, and J. Nishimura, Astrophys. J. 601, 340 (2004).

[13] A. W. Strong, E. Orlando, and T. R. Jaffe, Astron. Astrophys. 534, A54 (2011).

[14] Ł. Stawarz, V. Petrosian, and R. D. Blandford, Astrophys. J. 710, 236 (2010).

[15] I. V. Moskalenko and A. W. Strong, Astrophys. J. 493, 694 (1998); A. E. Vladimirov, S. W. Digel, G. JÃşhannesson, P. F. Michelson, I. V. Moskalenko, P. L. Nolan, E. Orlando, T. A. Porter, and A. W. Strong, Comput. Phys. Commun. 182, 1156 (2011).

[16] M. Aguilar et al., Phys. Rev. Lett. 121, 051102 (2018).

[17] R. A. Caballero-Lopez and H. Moraal, J. Geophys. Res. 109, A01101 (2004); L. Gleeson and W. Axford, Astrophys. J. 154, 1011 (1968). 